\title{
Minimal-Counterintuitiveness Revisited: Effects of cultural and ontological violations on concept memorability
}

\author{
Michaela Porubanova-Norquist, ${ }^{1}$ Daniel Joel Shaw ${ }^{2}$ \\ AND DiMITRIS XYGALATAS ${ }^{1}$
}

\author{
${ }^{1}$ LEVYNA Laboratory for the Experimental Research of Religion; \\ ${ }^{2}$ Social and Behavioural Neuroscience Research Group, CEITEC-Central \\ European Institute of Technology,
}

mise199@gmail.com,dan.shawj@gmail.com,Xygalatas@mac.com

Keywords

concept memory, cultural versus ontological expectations

\begin{abstract}
Many religious ideas have attributes that violate our expectations about the state of the natural world. It has been argued that minimal counter-intuitiveness (MCI), defined as a mild violation of innate (ontological) expectations, makes such ideas memorable and prone to cultural transmission. Empirical studies have examined memory for concepts that violate innate ontological expectations; however memorability of ideas that defy cultural or learned expectations have been (with few exceptions) overlooked. In our study, we compared memory for ideas that violate intuitive ontologies, learned expectations, and everyday, intuitive ideas. We discuss the mnemonic advantage of minimally counterintuitive ideas in terms of a combination of associative strength and bizarreness.
\end{abstract}

\section{Introduction}

In the field of the cognitive science of religion, it has been suggested that many cultural and more specifically religious ideas are "contagious" (Norenzayan and Atran 2004; Sperber 1996) and enjoy a cultural transmission advantage because they appeal to the architecture of human cognition (Boyer and Ramble 2001). One of the mechanisms involved in making religious ideas cognitively attractive seems to be related to attentional resources dedicated to those ideas, and consequently to their increased memorability (Boyer 1994, 2001). It has been argued that one aspect of religious concepts which makes them more memorable is that they are

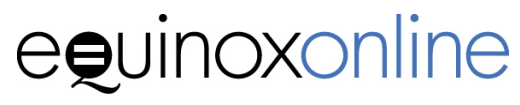


minimally counter-intuitive (MCI), i.e., they involve mild violations of our intuitive ontological expectations in the domains of folk physics, folk psychology, or folk biology (Barrett 2008; Boyer 2001; Gervais, Willard, Norenzayean and Henrich 2011; Norenzayean and Atran 2004). Such concepts were suggested to be more attention-grabbing and memorable, and possess greater inferential potential than other ideas (Barrett 2004). This constitutes a "cognitive optimum" (Boyer 1994, 2001), and these concepts are thus more likely to be transmitted. Indeed, minimally counterintuitive ideas seem to be abundant in the domain of religion (Barrett and Nyhof 2001; Boyer 2001; Norenzayan et al. 2006).

This attunement of memory to ontological violations has been observed in children (Banarjee, Haque and Spelke, in press) as well as adults (Barrett and Nyhof 2001; Boyer and Ramble 2001; Gonce et al. 2006; Norenzayan et al. 2006; Tweeney et al. 2006), using both immediate and delayed recall (Banarjee et al. in press; Barrett and Nyhof 2001; Gonce et al. 2006; Norenzayan et al. 2006); with narrative forms (Barrett and Nyhof 2001; Boyer and Ramble 2001) or concept lists (Gonce et al. 2006; Tweney et al. 2006). This is also congruent with an extensive psychological literature showing that bizarre or unexpected information has a mnemonic advantage due to its uniqueness when compared to purely mundane, intuitive information (Hunt 1995; Jacoby and Craik 1979; Schmidt 1991; Waddill and McDaniel 1998).

Pascal Boyer (1994) identified ontological categories, such as person, animal, plant and object (artificial or natural) as the domain concepts that possess certain physical, psychological, or biological attributes intuitively inherent in each category. Recognition of a particular object activates certain intuitive ontological expectations related to the object's attributes. Violating expectations that are inherent to the core knowledge based on ontologies is the mechanism that makes $\mathrm{MCI}$ concepts more salient and, in return, more memorable (Boyer and Ramble 2001; Norenzayam and Atran 2004).

Previous research in the cognitive science of religion has examined memory and the cognitive attractiveness of MCI ideas. Results have been somewhat inconsistent; while some studies suggested that MCI concepts are more memorable than purely intuitive ones (Banarjee et al. in press; Barrett and Nyhof 2001; Boyer and Ramble 2001), others dispute this assumption (Gonce et al. 2006; Upal et al. 2007), arguing instead that context is more crucial for the memorability of an idea (Upal 2010).

One line of studies compared the memorability of MCI ideas with those that are intuitive and maximally counterintuitive (i.e., those that violate two or more inferences; Atran and Norenyazan 2004; Banerjee et al. in press; Gonce et al. 2006; Harmon-Vukic, Upal and Sheenan 2012; Tweney et al. 2006; Upal 2010;

(C) Equinox Publishing Ltd. 2014

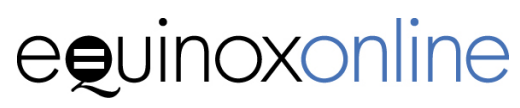


Upal et al. 2007). However, since many studies were focused on maximally counter-intuitive concepts and their memorability is rather well understood, we decided to focus on another aspect of concept memorability. In addition, we believe that the difference between MXCI and MCI concepts is one of quantity, merely amplifying the cognitive resources to be allocated to MXCI concepts, and thus making them more difficult to process and subsequently diminishing their memorability. Our focus therefore lied in memory for concepts quantitatively similar to MCI concepts, meaning that they do not impose greater cognitive demands to be remembered (such as in case of MXCI concepts), but with one important difference, that is violations of cultural conceptual knowledge which have been rather overlooked in the literature.

The main aim of our study was to understand how human attention and memory respond to violations of ontological versus cultural expectations. To this end we compared concepts that made similar demands on cognitive resources but varied fundamentally in terms of their quality. We used intuitive concepts as well as concepts involving two kinds of violations: those breaking intuitive ontological assumptions, and those violating culturally acquired assumptions. The latter can be understood as concepts that violate expectations with respect to their attributes, based upon our conceptual knowledge. Therefore, the fundamental distinction between intuitive and cultural expectation-violating concepts lies in the degree of their viability. Importantly, Iman and Richman (1991) showed that when processing time is limited, plausible bizarre information is remembered more than implausible bizarre information. Similarly, we do not expect a cat to start speaking because we have an intuitive understanding of ontology, and based on cats' biological properties the existence of this concept is impossible. In contrast, concepts like a wooden bottle or a blind driver violate only learned assumptions about the properties or abilities of individual concepts. Since these concepts are not impossible, we refer to them as minimally counter-schematic or cultural intuition-violating, as opposed to minimally counter-intuitive ones, which represent violation to ontological assumptions acquired early in ontogenesis (e.g., Spelke and Kinzler 2007)

To maximize internal validity we controlled for various potentially confounding variables. Some of the most important factors influencing attentional (and subsequently mnemonic) preference for certain information are word length, word frequency and latency of stimulus presentation. The word frequency effect refers to the finding that high frequency words are recalled to a greater extent than low frequency words (Hall 1954; Sumby 1963). The effect is opposite for recognition-i.e., low frequency words are better recognized than high frequency words (Gorman 1961). The word length effect refers to the superior immediate recall for lists of short compared with long words (Baddeley, Thomson, and

(C) Equinox Publishing Ltd. 2014

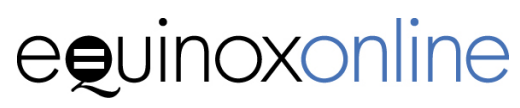


Buchanan 1975) due to the more efficient rehearsal of the former. Lastly, the effect of stimulus latency (or exposure time) on memory is also of relevance. The time dedicated to processing a certain stimulus correlates with the memory for that stimulus (for a review see Gouden and Nicolas 2012). Our study is the first to control for all those factors in order to afford precise measurements of the memorability of MCI concepts.

Furthermore, we examined memorability for agents versus non-agents through the inclusion of individual ontological categories as suggested by Boyer (1994). The sensitivity of the human mind towards clues related to agency (Barrett and Johnson 2003) suggests the evolutionary importance of detecting agency within the environment (Guthrie 1993). Interestingly, studies have demonstrated an over-attribution of agency to non-agents, clearly showing evidence for the existence of a "hyperactive agency detection device" in humans (Barrett 2000; see also Atran 2002; Guthrie 1993). To our knowledge, however, memory for agents in comparison to non-agents has not been investigated beyond the demonstration of agency attribution to non-agents via minimally counter-intuitive concepts (such as an "intelligent plant").

The experiment consisted of immediate memory recall and delayed recognition tasks for three different concept categories: intuitive, cultural expectationviolating, ontological expectation-violating (MCI). Furthermore, the presence or absence of agents in each category was included by following Boyer's four different ontological categories (human, animal, plant, object). The primary aim of the study was to understand the memory for MCI concepts when faced with intuitive concepts compared with those involving violations of "cultural intuitions."

\section{Method}

Participants studied 48 concepts comprising three concept categories: intuitive (INT), cultural expectation-violating (EVC), and ontological expectation-violating (MCI). Memory for individual concepts was assessed twice, immediately after the initial learning phase and subsequently a month after.

Subjects. Fifty Czech undergraduate and graduate students (28 female, 22 male; age range: 20-23) participated in the study in exchange for class credit. No subjects reported memory pathologies, and all reported normal or correctedto-normal vision.

Stimuli and apparatus. Forty-eight concepts were constructed for the purpose of the experiment. Each concept consisted of an adjective and a noun. Word frequency and word length were controlled within and across all three concept categories. All concepts were presented in Czech. The experiment was programmed on MATLAB version 19.0, and carried out on a Fujitsu laptop with a

(C) Equinox Publishing Ltd. 2014

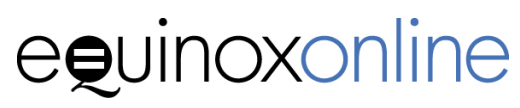


17-inch colour monitor.

The concepts were created as follows: each concept represented one concept category and one ontological category. Three different concept categories were presented:

1. Intuitive concepts (16 concepts), e.g., plastic clock

2. Ontological expectation-violating concepts (i.e., minimally counterintuitive; 16 concepts), e.g., talking cat

3. Cultural expectation-violating concepts (16 concepts), e.g. illiterate teacher

Within each concept category, four different ontological categories were included: human, animal, plant and objects.

Procedure. Prior to the experiment, subjects were told that the study concerned learning of concepts and their task would be to study all the presented concepts. The experiment consisted of two separate parts. A basic concepts learning paradigm was used, such that each subject was administered 48 concepts (16 in each category), presented randomly. Each concept was presented in white letters on a black screen individually for $1500 \mathrm{~ms}$ with a $500 \mathrm{~ms}$ interstimulus interval. After the initial learning phase, a filler task was presented for 2 mins requiring the subject to solve a range of mathematical operations comprised of simple additions, subtractions, multiplication, and division. Afterwards, subjects were prompted to recall as many concepts as possible, including both the adjective and noun, with a time limit of one minute (in general, subjects reported that time was sufficient for the recall). This sequence (learning phase, filler task, recall) was repeated in order to observe the permeability of the concepts in memory, i.e., the effect of repeated exposure on the recall of individual concepts.

In order to determine the pervasiveness of individual concepts in long-term term memory, participants were contacted one month after the initial learning phase took place. All 50 participants responded promptly and were presented with a surprise recognition test. In the task, 48 old and 48 new concepts were presented to the participants. Their task was to indicate which concepts were presented in the initial study.

The scoring of the recalled items was as following: an item was considered as correctly recalled only in the case of precise recall of both adjective and noun. Recall was scored by two independent raters. The same scoring process was applied for the recognition task, with the only difference of discounting of any false alarms of newly presented items. 
Results

We examined memory for expectation violation of various ontological categories with repeated-measures ANOVA, incorporating concept category (EVC, MCI, INT), ontological category (human, animal, plant, object), and sequence (recall 1, recall 2, one-month post-experiment recognition) as within-subject variables.

The descriptive statistics for individual variables is displayed in Table 1.

\section{Overall recall}

\section{Ontological category}

Human

Animal

Plant

Object

\section{Concept category}

EVC

$\mathrm{MCI}$

INT
Mean (SD)

Table 1. Means (standard deviations) for recall of individual concepts based on ontological or concept category

We observed a main effect of concept category, $F(2,94)=36.51, p<.001$, $\eta_{\mathrm{p}}{ }^{2=} .44$, ontological category, $F(3,141)=7.37, p<.001, \eta_{\mathrm{p}}{ }^{2}=.14$, and sequence, $F(2,94)=333.24, p<.001, \quad \eta_{\mathrm{p}}^{2}=.88$. We also found a significant interaction between concept category and sequence, $F(4,188)=13.71, p<.001, \eta_{\mathrm{p}}{ }^{2=} .23$. The directions of these effects are given below.

Concept category. Participants throughout all memory tests remembered more EVC concepts $(\mathrm{M}=5.31, \mathrm{SD}=.1 .40)$ compared with INT $(\mathrm{M}=3.65, \mathrm{SD}=1.35)$, $t(49)=7.28, p<.001$, and $\mathrm{MCI}$ concepts $(\mathrm{M}=3.78, \mathrm{SD}=1.48), t(49)=7.66, p<.001$. No differences between the recall of INT and MCI concepts were found.

Ontological category. As depicted in Figure 1, the recall of concepts in the ontological domain human $(\mathrm{M}=1.25, \mathrm{SD}=.38)$ were recalled to a greater extent than all three other ontological categories: animal $(\mathrm{M}=1.06, \mathrm{SD}=.43)$, $t(49)=3.06, p<.001$; plant $(\mathrm{M}=0.99, \mathrm{SD}=.42), t(49)=4.27, p<.001$ and object $(\mathrm{M}=.95, \mathrm{SD}=.41), t(49)=4.01, p<.001$. No significant differences were observed among the three non-human ontological categories in terms of recall.

(C) Equinox Publishing Ltd. 2014

please check the puctuation for these paragraphs 


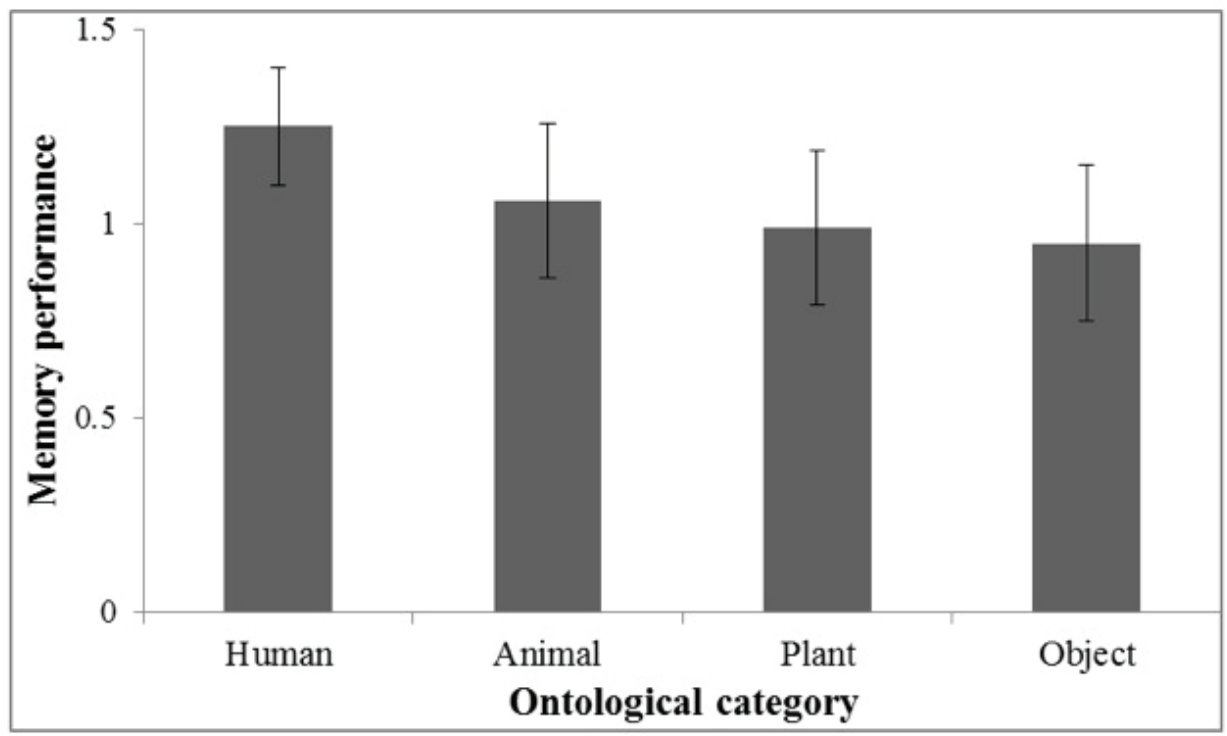

Figure 1. Memory performance for individual ontological categories (error bars represent standard deviations).

Test phase. Unsurprisingly, participants recalled more items in the last recognition test $(\mathrm{M}=8.51, \mathrm{SD}=2.46)$ than in the previous first and second immediate recall tests $(\mathrm{M}=1.77, \mathrm{SD}=.81, t(49)=20.83, p<.001 ; \mathrm{M}=2.60, \mathrm{SD}=.72)$, $t(49)=17.15, p<.001$ respectively).

Interestingly, in terms of the interaction between concept category and sequence, in the first two immediate recalls EVC concepts were recalled better $(\mathrm{M}=2.95, \mathrm{SD}=1.13)$ than $\mathrm{INT}$ concepts $(\mathrm{M}=2.10, \mathrm{SD}=.98), t(49)=3.95, p<.001$; and MCI concepts $(\mathrm{M}=1.49, \mathrm{SD}=1.03), t(49)=7.23, p<.001$. The recall of INT concepts was superior to recall of MCI concepts, $t(49)=3.24, p<.01$.

However, the follow-up recognition test showed that the recall of both EVC and $\mathrm{MCI}$ concepts $(\mathrm{M}=10.14, \mathrm{SD}=2.55 ; t(48)=8.66, p<.001 ; \mathrm{M}=8.53, \mathrm{SD}=$ $3.30, t(48)=4.10, p<.001$, respectively, $)$ was superior to INT concepts $(\mathrm{M}=6.86$, $\mathrm{SD}=2.86)$. We still found a memory advantage for EVC over MCI concepts, $t(48)=4.22, p<.001$. See Figure 2 for memory performance in individual memory tasks (recall, recognition, and overall performance).

\section{Discussion}

The aim of this study was to explore the memorability of two different kinds of expectation-violating concepts. The results showed that in terms of concept category, concepts violating cultural expectations were better recalled in both

(C) Equinox Publishing Ltd. 2014 


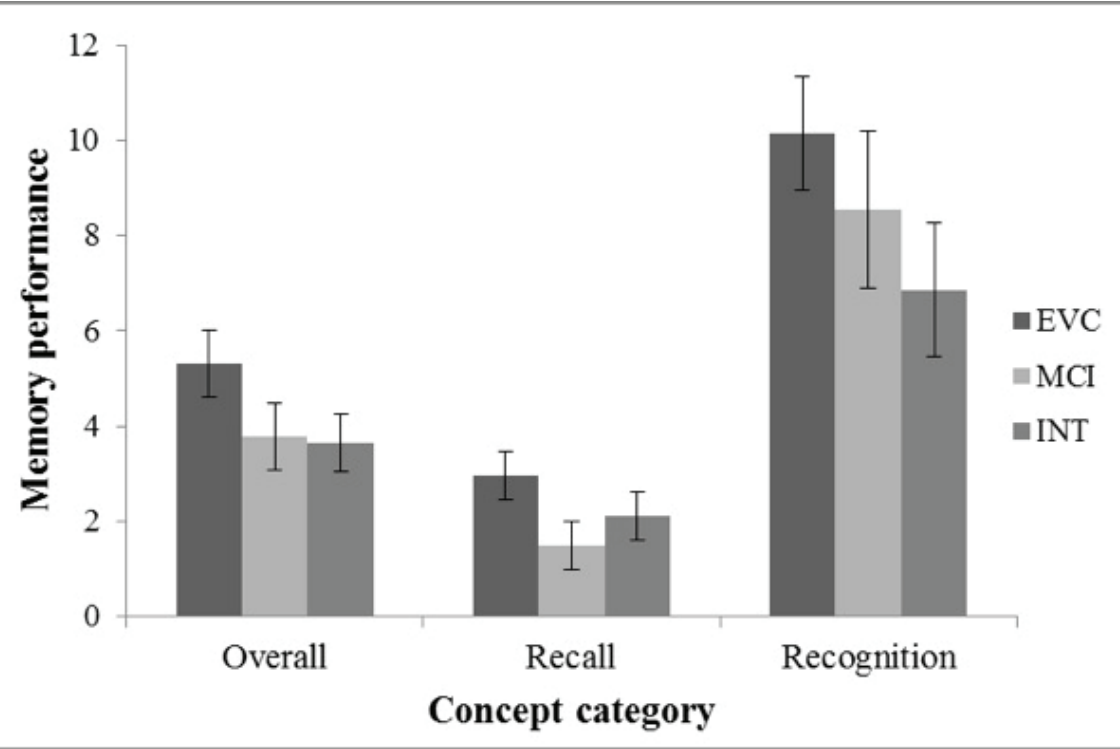

Figure 2. Memory performance for individual concept categories illustrating the overall, recall, and recognition memory performance (error bars represents standard deviations).

immediate and delayed memory tests, while concepts violating ontological expectations were better recalled in delayed recognition test when compared to intuitive concepts. Secondly, agency was an important determinant of concept memorability, since human agents in particular were resistant to forgetting compared to other ontological categories (animals, plants, and objects).

Based on our results, it seems that human memory is attracted primarily to ideas that violate cultural expectancies, i.e., ideas that could potentially exist, but are not typically encountered in everyday interactions with the world (see Apendix for examples). On the other hand, ideas that violate intuitive expectancies, such as minimally counter-intuitive ideas, do not seem to enjoy an immediate memory advantage, although they are remembered better than intuitive, everyday ideas after a delay — in our study, a month after the initial presentation. Those results are in accordance with those of other studies that found minimally counterintuitive ideas persistent in memory through a delayed recall test (Boyer and Ramble 2001; Norenzayan et al. 2006).

Expectation-violating concepts have two main characteristics: not only are they slightly bizarre - and we would expect the effects of bizarreness or distinctiveness to yield better memory for those items (Hunt 1995; Ranganath and Rainer 2003; Schmidt 1991; Waddill and McDaniel 1998) — they also refer to

(C) Equinox Publishing Ltd. 2014 
the strength of the cultural associations between the adjective and the noun. For instance, consider the concept illiterate teacher: there is a strong association between being a teacher and being intelligent, or at least capable of reading and writing. This is almost a prototypical attribute of a teacher, thus the association has great strength, i.e., increased memorability. Violating this prominent attribute seems to strengthen memorability by boosting its distinctiveness/bizarreness. In the free recall test, searching for free associations between a noun and the respective adjective first produces associations that are appropriate, and thus the associative strength is high. In our case, the adjective-noun associative strength refers to the crucial attribute of the noun, but in a rather unexpected or somewhat contrary way. This, we suggest, enhances recall due to the bizarreness. Therefore, those unexpected cultural associations that occur at the adjective-noun level might serve as "mnemonic devices" resulting in greater recall for cultural expectation-violating concepts than for minimally counter-intuitive or intuitive words.

A second important finding relates to memory for individual ontological categories as suggested by Boyer (1994). Throughout all concept categories, concepts pertaining to humans were recalled better than concepts related to other, non-human ontological categories (animal, plant, object). It is not surprising that a memory preference for agents, particularly humans, was observed. First of all, humans have a tendency to detect agency even where there is none, especially in dubious situations (Barrett and Johnson 2003; Gray and Wegner 2010; Gurthrie 1980, 1993; Heider and Simmel 1944; Lawson and McCauley 1990; Michotte 1962; Scholl and Tremoulet 2000). This has led Justin Barrett to argue for the existence of a dedicated mechanism which he termed a "hyperactive agency detection device" $(2000 ; 2004)$. This tendency to over-detect agency has an adaptive function, allowing us to avoid potential misses that might threaten our survival. Similarly, information regarding agents motivates increased learning. For example, mechanisms related to the detection of cheating might enhance memory for incidents of deception in order to avoid potential future mistreatment (Bell and Buchner 2009; Mealey, Daood, and Krage 1996). A recent study by Chang and Safley (2009) demonstrated that memory in situations of social interaction is predominantly guided by a general expectation-violation system. Therefore, it seems that human agents that have violated our expectations are particularly attention-grabbing.

We believe that our study brings important insights for understanding the role of counter-intuitiveness in memory, and potentially cultural transmission. By limiting cognitive resources that could be allotted to individual concepts, we show that both expectation-violating concept categories (MCI and EVC) enjoy a memory advantage.

(C) Equinox Publishing Ltd. 2014

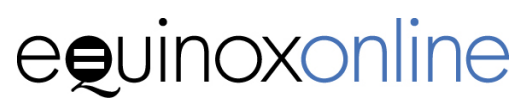


To conclude, based on our findings, it seems that concepts involving humans as well as ideas violating our expectations are cognitively more attractive, potentially due to the fact that they serve as opportunities for conceptual learning that prevent individuals from facing potential threats to their survival.

\section{References}

Atran, S. 2002. In Gods We Trust: The Evolutionary Landscape of Religion. New York: Oxford University Press.

Atran, S., and Norenzayan, A. 2004. "Religion's evolutionary landscape: Counterintuition, commitment, compassion, communion." Behavioral and Brain Sciences 27: 713-770. http://dx.doi.org/10.1017/S0140525X04000172

Baddeley, A. D., N. Thomson and M. Buchanan. 1975. "Word length and the structure of short-term memory." Journal of Verbal Learning and Verbal Behavior 14: 575-589. http://dx.doi.org/10.1016/S0022-5371(75)80045-4

Banerjee, K., O.S. Haque and E. S. Spelke. in press. "Melting lizards and crying mailboxes: Children's preferential recall of minimally counterintuitive concepts." Cognitive Science.

Barrett, J. L. 2000. "Exploring the natural foundations of religion." Trends in Cognitive Sciences 4: 29-34. http://dx.doi.org/10.1016/S1364-6613(99)01419-9 2004. Why would anyone believe in god? Lanham, MD: AltaMira Press. 2008. "Coding and Quantifying Counterintuitiveness in Religious Concepts: Theoretical and Methodological Reflections." Method and Theory in the Study of Religion 20: 308-338. http://dx.doi.org/10.1163/157006808X371806 and A. H. Johnson. 2003. "The role of control in attributing intentional agency to inanimate objects." Journal of Cognition and Culture 3(3): 208-217. http:// dx.doi.org/10.1163/156853703322336634

and M. Nyhof. 2001. "Spreading non-natural concepts." Journal of Cognition and Culture 1: 69-100. http://dx.doi.org/10.1163/156853701300063589

Bell, R., and A. Buchner. 2009. "Enhanced source memory for names of cheaters." Evolutionary Psychology 7(2): 317-330.

Boyer, P. 1994. The Naturalness of Religious Ideas: A Cognitive Theory of Religion. Berkeley: University of California Press.

2001. Religion Explained: The Evolutionary Origins of Religious Thought. New York: Basic Books.

and C. Ramble. 2001. "Cognitive templates for religious concepts: Cross-cultural evidence for recall of counter-intuitive representations." Cognitive Science 25: 535-564. http://dx.doi.org/10.1207/s15516709cog2504_2

Gervais, W. M., A. K. Willard, A. Norenzayean and J. Henrich. 2011. "The cultural transmission of faith: Why innate intuitions are necessary, but insufficient, to explain religious belief." Religion 41(3): 389-410. http://dx.doi.org/10.1080/0 048721X.2011.604510

(C) Equinox Publishing Ltd. 2014

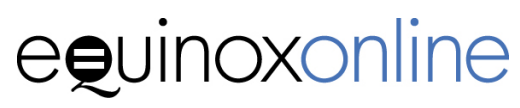


Gonce, L., M.A. Upal, D.J. Slone and R.D. Tweney. 2006. "Role of context in the recall of counterintuitive concepts." Journal of Cognition and Culture 6(3-4): 521-547. http://dx.doi.org/10.1163/156853706778554959

Gorman, A. M. 1961. "Recognition memory for nouns as a function of abstractedness and frequency." Journal of Experimental Psychology 61: 23-39. http://dx.doi. org $/ 10.1037 / \mathrm{h} 0040561$

Gouden, Y., and S. Nicolas. 2012. "The impact of processing time on the bizarreness and orthographic distinctiveness effects." Scandinavian Journal of Psychology 53(4): 287-294. http://dx.doi.org/10.1111/j.1467-9450.2012.00945.x

Gray, K. and D. Wegner. 2010. "Blaming God for Our Pain: Human Suffering and the Divine Mind." Personality and Social Psychology Review 14(1): 9-10. http:// dx.doi.org/10.1177/1088868309350299

Guthrie, S. 1980. "A cognitive theory of religion." Current Anthropology 21: 181-203. 1993. Faces in the Clouds: A New Theory of Religion. Oxford: Oxford University Press. http://dx.doi.org/10.1086/202429

Hall, J. 1954. "Learning as a function of word-frequency." American Journal of Psychology 67: 138-140. http://dx.doi.org/10.2307/1418080

Harmon-Vukic, M., M.A. Upal and K.J. Sheenan. 2012. "Understanding the memory advantage of counterintuitive concepts." Brain, Religion, and Behavior 2(2): 121-139. http://dx.doi.org/10.1080/2153599X.2012.672816

Heider, F., and M. Simmel. 1944. "An experimental study of apparent behaviour." American Journal of Psychology 13: 243-259. http://dx.doi.org/10.2307/1416950

Hunt, R.R. 1995. "The subtlety of distinctiveness: What von Restorff really did." Psychonomic Bulletin and Review 2: 105-112. http://dx.doi.org/10.3758/BF03214414

Iman, S. and C. L. Richman. 1991. "Is the bizarreness effect a special case of sentence reorganization?" Bulletin of the Psychonomic Society 29: 429-432. http:// dx.doi.org/10.3758/BF03333962

Jacoby, L.L., and F.I.M. Craik. 1979. "Effects of elaboration of processing at encoding and retrieval: Trace distinctiveness and recovery of initial context." In Levels of processing and human memory, edited by L. S. Cermak and F. I. M. Craik, 1-21. Hitlsdale, NJ: Lawrence Ertbaum Associates.

Lawson, E T., and R.N. McCauley. 1990. Rethinking Religion: Connecting Cognition and Culture. Cambridge: Cambridge University Press.

Mealey, L., C. Daood and M. Krage. 1996. "Enhanced memory for faces of cheaters." Ethology and Sociobiology 17: 119-128. http://dx.doi.org/10.1016/01623095(95)00131-X

Michotte, A. 1962. The perception of causality. Andover, MA: Methuen.

Norenzayan, A., and S. Atran. 2004. "Cognitive and emotional processes in the cultural transmission of natural and nonnatural beliefs." In The Psychological Foundations of Culture, edited by M. Schaller and C. Crandall, 149-169. Hillsdale, NJ: Lawrence Erlbaum Associates. 
S. Atran, J. Faulkner and M. Schaller. 2006. "Memory and mystery: The cultural selection of minimally counterintuitive narratives." Cognitive Science 30: 531-553. http://dx.doi.org/10.1207/s15516709cog0000_68

Ranganath C., and G. Rainer. 2003. "Neural mechanisms for detecting and remembering novel events." Nature Review Neuroscience 4: 193-202. http://dx.doi. org/10.1038/nrn1052

Schmidt, S. R. 1991. "Can we have a distinctive theory of memory?" Memory and Cognition 19: 523-542. http://dx.doi.org/10.3758/BF03197149

Scholl, B. J., and P. D. Tremoulet. 2000. "Perceptual causality and animacy." Trends in Cognitive Sciences 4(8): 299-309. http://dx.doi.org/10.1016/S13646613(00)01506-0

Spelke, E. S., and K. D. Kinzler. 2007. “Core knowledge.” Developmental Science 10: 89-96. http://dx.doi.org/10.1111/j.1467-7687.2007.00569.x

Sperber, D. 1996. Explaining Culture: A Naturalistic Approach. Oxford: Blackwell.

Sumby, W. H. 1963. "Word frequency and serial position effects." Journal of Verbal Learning and Verbal Behavior 1: 443-450. http://dx.doi.org/10.1016/S00225371(63)80030-4

Tweney, R. D., M. A. Upal, L. O. Gonce, D. J. Slone and K. Edwards. 2006. "The creative structuring of counterintuitive worlds." Journal of Cognition and Culture 6(3-4): 3-4.

Upal, A. M. 2010. "An alternative view of the minimal counter-intuitiveness effect." Journal of Cognitive Systems Research 11(2): 194-203. http://dx.doi. org/10.1016/j.cogsys.2009.08.003

L. O. Gonce, R. D. Tweney and D. J. Slone. 2007. “Contextualizing counterintuitiveness: How context affects comprehension and memorability of counterintuitive concepts." Cognitive Science 31(3): 415-439. http://dx.doi. org/10.1080/15326900701326568

Waddill, P. J, and M. A. McDaniel. 1998. "Distinctiveness effects in recall: Differential processing or privileged retrieval." Memory and Cognition 26(1): 108-120. http://dx.doi.org/10.3758/BF03211374

(C) Equinox Publishing Ltd. 2014

\section{equinoxonline}

